\title{
Hepatitis E seroprevalence and associated risk factors in Croatian liver transplant recipients
}

\author{
Anna Mrzljak ${ }^{[1],[2],}$ Petra Dinjar-Kujundzic ${ }^{[1]}$, Tatjana Vilibic-Cavlek ${ }^{[2],[3]}$, Lorena Jemersic ${ }^{[4]}$, \\ Jelena Prpic ${ }^{[4]}$, Oktavija Dakovic-Rode ${ }^{[5],[6], ~ B r a n k o ~ K o l a r i c}{ }^{[7],[8]}$ and Adriana Vince ${ }^{[2],[9]}$
}

[1]. University Hospital Merkur, Department of Gastroenterology, Zagreb, Croatia.

[2]. University of Zagreb, School of Medicine, Zagreb, Croatia.

[3]. Croatian Institute of Public Health, Department of Virology, Zagreb, Croatia.

[4]. Croatian Veterinary Institute, Zagreb, Croatia.

[5]. University Hospital for Infectious Diseases "Dr Fran Mihaljevic", Department of Clinical Microbiology, Division of Medical Virology, Zagreb, Croatia.

[6]. University of Zagreb, School of Dental Medicine, Zagreb, Croatia.

[7]. Andrija Stampar Teaching Institute of Public Health, Zagreb, Croatia.

[8]. University of Rijeka, Faculty of Medicine, Rijeka, Croatia.

[9]. University Hospital for Infectious Diseases "Dr Fran Mihaljevic",

Department for Viral Hepatitis, Zagreb, Croatia.

\begin{abstract}
Introduction: Solid-organ transplant recipients are at risk of hepatitis E virus (HEV) infection. We analyzed the seroprevalence/ risk factors of HEV in Croatian liver transplant recipients. Methods: Two hundred forty-two serum samples were tested for HEV immunoglobuline IgG/IgM and HEV RNA. Sociodemographic data and risk factors were collected using a questionnaire. Results: HEV IgG seroprevalence rate was $24.4 \%$. Positive/equivocal HEV IgM were found in two patients. HEV RNA was not detected. Logistic regression showed that older age, female gender, rural area/farm, water well, and septic tank were associated with HEV seropositivity. Conclusions: This study revealed a high exposure rate to HEV in Croatian liver recipients.
\end{abstract}

Keywords: Hepatitis E virus. Seroprevalence. Risk factors. Liver transplant recipients. Croatia.

Hepatitis E virus (HEV) infection rate is increasing in several European countries, including reports of infection in immunocompromised patients, who may develop chronic HEV infections with rapid progression of disease and cirrhosis, after solid-organ transplantation $(\mathrm{SOT})^{1}$. Zoonotic genotypes 3 and 4 are endemic in Europe and hyperendemic in Southern France where foodborne transmissions are most common, primarily due to consumption of raw and undercooked meat of domestic pigs, wild boar, and deer².

Epidemiological studies from Europe estimate that HEV seroprevalence among SOT recipients differs widely, ranging from $2.7 \%$ to greater than $43 \%{ }^{3,4}$. The large heterogeneity

Corresponding author: Anna Mrzljak

e-mail: anna.mrzljak@mef.hr

Orcid: 0000-0001-6270-2305

Received 15 June 2019

Accepted 18 July 2019 in seropositivity may be partially due not only to regional differences but also to differences in the sensitivity and specificity of immunoassays used to detect HEV antibodies. Data on HEV epidemiology, routes of transmission, and associated risk factors in solid-organ transplant cohorts in Southeast Europe are largely unknown.

Croatia has an increasing transplant population, which according to the International Registry in Organ Donation and Transplantation is estimated to be 28.2 liver transplantations (LTs) per million population, one of the highest LT rates in the world currently ${ }^{5}$. In Croatia, HEV seroprevalence in different population groups (alcohol abusers, war veterans, injecting drug users, and healthcare professionals) is estimated to be $8.9 \%$, $8.6 \%, 6.1 \%$, and $2.7 \%$, respectively ${ }^{6}$.

This cross-sectional study aimed to determine the HEV seroprevalence and the possible sociodemographic and risk factors associated with HEV infection in a large cohort of Croatian liver transplant recipients. 
The study was conducted from June to October 2017 and included 242 adult liver recipients, transplanted from 1994 to 2013 at the Liver Transplant Centre, Merkur University Hospital (Zagreb, Croatia), which is a high-volume transplant center with approximately 120 performed liver transplants yearly, representing over $90 \%$ of LTs in the country. All participants received the same immunosuppressive protocol, comprising calcineurin inhibitors, steroids (discontinued after 3 months), and mycophenolate mofetil, after transplantation. The sociodemographic data and potential risk factors were collected by interviewing all the participants using a questionnaire. Medical data were collected from patients' medical records.

Serum samples were tested for the presence of HEV antibodies and HEV ribonucleic acid (RNA) during routine visits to the outpatient transplant clinic. All samples were initially tested for the presence of HEV immunoglobulin G ( $\operatorname{IgG}$ ) antibodies. $\operatorname{IgG}$ reactive samples were further tested for the presence of HEV immunoglobulin M (IgM) antibodies to confirm/rule out recent infection. Serological testing was performed using commercial enzyme immunoassays (recomWell HEV IgG/IgM, Mikrogen, Neuried, Germany) and using highly purified HEV open reading frame - ORF2 (genotype 1 and genotype 3) antigens. Manufacturer reveals a diagnostic sensitivity of $98.9 \%$ and a specificity of $98.5 \%$. HEV seroprevalence rate prior to the transplantation was unknown.

Additionally, all samples were tested for the presence of HEV RNA. RNA extraction was performed using a QIAamp viral RNA extraction kit (Qiagen, Hilden, Germany), whereas reverse transcription was performed using the GoScript Reverse Transcription System for reverse transcription polymerase chain reaction (RT-PCR) (Promega, Madison, USA) in a GeneAmp PCR System 9700 machine (Applied Biosystems, USA). All samples were tested using a real-time PCR that targets an RNA sequence within the highly conserved ORF3 genome region.

The study was approved by the Hospital Ethics Committee. Each patient provided written informed consent for inclusion in the study.

Statistical analysis was performed using Stata/IC ver. 14.2 (StataCorp LLC). Normality of distributions was assessed using Shapiro-Wilk test. Differences between the groups were tested using chi-squared and Fischer's exact tests (categorical variables) or Mann-Whitney U and Kruskal-Wallis tests (ordinal or numerical variables). The association between the HEV seroprevalence and potential risk factors was tested using univariate and multiple logistic regression models. Level of statistical significance was set at $\alpha=0.05$.

HEV IgG antibodies were detected in 59/242 (24.4\%) of transplant patients after LT. The median time after transplant was 5 years (range, 3-14 years). HEV IgM antibodies were noted in one patient, and one patient showed equivocal result. None of the tested sample was positive for HEV RNA, excluding acute, but more importantly chronic hepatitis E infection, which may develop in immunocompromised population and lead to graft loss.

There were no differences between the anti-HEV IgGpositive and IgG-negative groups regarding the etiology of liver disease, area of residence, profession, and eating habits. Anti-HEV IgG-positive patients were older (median 62 vs. 59 years, $\mathrm{p}=0.010)$ and more predominantly female $(37.3 \%$ vs. $18.6 \%, \mathrm{p}=0.003$ ) and had more household sewage systems connected to a septic tank compared to anti-HEV IgGnegative patients $(55.6 \%$ vs. $38.1 \%, \mathrm{p}=0.027)$. In a group of anti-HEV IgG-seropositive patients, $88.9 \%$ had primary or lower secondary education compared to $75.0 \%$ in a group of anti-HEV-seronegative patients $(\mathrm{p}=0.024)$ (Table 1). According to a univariate logistic regression model, factors associated with anti-HEV IgG seropositivity in transplant patients were female gender (odds ratio [OR], 2.61; 95\% confidence interval [CI], 1.42-4.81), older age (with each year's increase in age) (OR, 1.05; 95\% CI, 1.02-1.09), and a household sewage system connected to a septic tank (OR, 2.03; 95\% CI, 1.09-3.78) (Table 2). After adjusting for age and gender, a rural area of residence (adjusted OR [AOR], 2.17; 95\% CI, 1.10-4.27), a farm within a household (AOR, 2.79; 95\% CI, 1.31-5.92), use of well as the source of drinking water (AOR, 3.09; 95\% CI, 1.11-8.57), and sewage system connected to a septic tank (AOR, 3.38; 95\% CI, 1.64-6.95) were associated anti-HEV IgG seroprevalence. Production and consumption of cured and undercooked meat, occupational exposure, and the number of household members were not significantly associated with anti-HEV IgG seropositivity (Table 2). In the multiple logistic regression model, independent risk factors for anti-HEV IgG seropositivity were female gender (OR, 4.13; 95\% CI, 2.03-8.37), older age (OR, 1.05; 95\% CI, 1.01-1.09), and sewage system connected to a septic tank (AOR, 3.38; 95\% CI, 1.64-6.95), whereas the highest level of education was identified as a protective factor (OR, $0.06 ; 95 \%$ CI, 0.01-0.54) (Table 3).

This is the first cross-sectional study assessing the anti-HEV IgG seroprevalence and associated risk factors in Croatian liver transplant patients. The results of this study showed a prevalence rate of $24.4 \%$ in transplant cohorts, suggesting a high degree of previous exposure to HEV.

Studies on anti-HEV IgG seroprevalence in liver recipients in Europe reported variable prevalence rates. Moderate prevalence rates have been found in Germany $(4 \%)^{7}$ and Spain $(2.7 \%)^{3}$. Posttransplant anti-HEV IgG prevalence rate in Croatian patients is consistent to those reported from France, which has the highest HEV seroprevalence rates in Europe ranging up to $29 \%$ in adult liver recipients ${ }^{8}$. Variations in HEV seroprevalence between countries may reflect not only the differences in viral circulation within a geographical region but also the impact of assays used in particular studies; therefore, the data should be cautiously interpreted.

Results of this study showed that female gender, older age, rural type of living area, farm within a household, water well as a supply system, and a septic tank within a household sewage system were associated with anti-HEV IgG seropositivity in the univariate risk analysis. On the contrary, the highest educational level and a recent travelling experience as markers for a higher socioeconomic status were inversely related with HEV seropositivity. A similar inverse correlation between HEV seropositivity rates and the level of education was observed in other studies? 
TABLE 1: Seroprevalence of hepatitis E virus in Croatian liver transplant recipients according to sociodemographic characteristics and risk factors.

\begin{tabular}{|c|c|c|c|}
\hline Characteristics & HEV IgG negative N (\%) & HEV IgG positive $\mathrm{N}(\%)$ & p \\
\hline Gender & & & 0.003 \\
\hline Male & $136(81.4)$ & $31(18.6)$ & \\
\hline Female & $47(62.7)$ & $28(37.3)$ & \\
\hline Age at sampling, yrs median & $59(32-80)$ & $62(40-75)$ & 0.010 \\
\hline Hepatitis B or C & $39(21.3)$ & $10(16.9)$ & \\
\hline Alcoholic cirrhosis & $94(51.4)$ & $28(47.5)$ & \\
\hline Autoimmune cirrhosis & $19(10.4)$ & $9(15.3)$ & \\
\hline Metabolic diseases & $8(4.4)$ & 0 & \\
\hline Cryptogenic liver disease & $12(6.6)$ & $8(13.6)$ & \\
\hline Educational level* & & & 0.024 \\
\hline ISCED 1 & $37(22.0)$ & $18(33.3)$ & \\
\hline ISCED 2 & $89(53.0)$ & $30(55.6)$ & \\
\hline ISCED 3-4 & $13(7.7)$ & $5(9.3)$ & \\
\hline ISCED 5-6 & $29(17.3)$ & $1(1.9)$ & \\
\hline \multicolumn{4}{|l|}{ Number of household members } \\
\hline 1 & $14(8.3)$ & $3(5.6)$ & \\
\hline $2-4$ & $127(75.6)$ & $41(75.9)$ & 0.809 \\
\hline 5 or more & $26(15.5)$ & $10(18.5)$ & \\
\hline Type of living area & & & 0.159 \\
\hline \multicolumn{4}{|l|}{ Source of drinking water } \\
\hline Public water supply system & $134(79.8)$ & $37(68.5)$ & \\
\hline Water tank & $1(0.6)$ & $1(1.9)$ & \\
\hline Water well & $13(7.7)$ & $8(14.8)$ & 0.249 \\
\hline Bottled water & $13(7.7)$ & $7(13)$ & \\
\hline Natural spring & $7(4.2)$ & $1(1.9)$ & \\
\hline \multicolumn{4}{|l|}{ Sewage system } \\
\hline Public sewage system & 10461.9 & $24(44.4)$ & \multirow{2}{*}{0.027} \\
\hline Septic tank & $64 \quad 38.1$ & $30(55.6$ & \\
\hline Travelling history (within the last 3 years) & $57 \quad 33.9$ & $9(16.7)$ & 0.017 \\
\hline
\end{tabular}

*The International Standard Classification of Education (ISCED): 1, primary education; 2, lower secondary education; 3-4, upper and postsecondary nontertiary education; 5-6, tertiary education.

A slightly higher seroprevalence rate in females was also reported in a previous Croatian study ${ }^{6}$, while some European studies reported higher seropositivity in males ${ }^{10}$. The metaanalysis on 73 European anti-HEV IgG seroprevalence studies showed that 45 of the 73 studies had no significant differences in HEV seroprevalence between genders ${ }^{11}$.

Consistent with other seroepidemiological studies ${ }^{11}$, our results showed that older age was associated with higher seroprevalence rates, suggesting a lifelong cumulative exposure to the virus.

Rural area of residence was reported as a risk factor for HEV seropositivity in several studies ${ }^{6,12}$. Moreover, living in a rural area with a farm was associated with HEV seropositivity in our cohort of transplant patients. Croatian data show high HEV seroprevalence rates (up to 91.7\%) and HEV RNA prevalence rate of $13.3 \%$ in domestic pigs $^{13}$. The rural population is more 
TABLE 2: Univariate logistic regression analysis of risk factors associated with anti-hepatitis $E$ virus immunoglobulin $G$ seropositivity among liver transplant recipients.

\begin{tabular}{|c|c|c|c|c|}
\hline Exposure group/variables & OR & OR $95 \% \mathrm{Cl}$ & AOR $^{a}$ & AOR $95 \% \mathrm{Cl}$ \\
\hline Sex (female vs. male) & 2.61 & $1.42-4.81$ & & \\
\hline Time after transplant, yrs & 0.95 & $0.86-1.05$ & 0.93 & $0.84-1.03$ \\
\hline Hepatitis B or C & Ref & & Ref & \\
\hline Alcoholic cirrhosis & 1.16 & $0.51-2.61$ & 1.32 & $0.57-3.08$ \\
\hline Autoimmune cirrhosis & 1.84 & $0.64-5.30$ & 1.88 & $0.58-6.04$ \\
\hline Cryptogenic liver disease & 1.42 & $0.37-5.41$ & 1.27 & $0.30-5.39$ \\
\hline Geographic region (coastal vs. continental) & 1.02 & $0.55-1.92$ & 1.17 & $0.611-2.24$ \\
\hline \multicolumn{5}{|l|}{ Educational level (ISCED) ${ }^{* *}$} \\
\hline ISCED 1 & Ref & & Ref & \\
\hline ISCED 2 & 0.69 & $0.35-1.39$ & 0.72 & $0.33-1.53$ \\
\hline ISCED 3-4 & 0.79 & $0.24-2.56$ & 1.06 & $0.31-3.63$ \\
\hline $2-4$ & 1.51 & $0.41-5.05$ & 1.34 & $0.35-5.2$ \\
\hline 5 or more & 1.80 & $0.42-7.61$ & 1.60 & $0.35-7.30$ \\
\hline Type of living area (rural vs. urban) & 1.64 & $0.88-3.05$ & 2.17 & $1.10-4.27$ \\
\hline Farm within a household (yes vs. no) & 1.83 & $0.93-3.60$ & 2.79 & $1.31-5.92$ \\
\hline Consumption of cured meat products (yes vs. no) & 0.95 & $0.47-1.92$ & 1.43 & $0.66-3.09$ \\
\hline Occupational exposure (yes vs.no) & 0.98 & $0.39-2.46$ & 1.76 & $0.66-4.69$ \\
\hline \multicolumn{5}{|l|}{ Source of drinking water } \\
\hline Public water supply system & Ref & & Ref & \\
\hline Water tank & 3.62 & $0.22-59.29$ & 4.17 & $0.24-71.58$ \\
\hline Water well & 2.23 & $0.86-5.78$ & 3.09 & $1.11-8.57$ \\
\hline Bottled water & 1.95 & $0.73-5.24$ & 1.23 & $0.43-3.57$ \\
\hline
\end{tabular}

aEach model adjusted for age and gender; AOR, adjusted odds ratio. *Referent. **The International Standard Classification of Education (ISCED): 1, primary education; 2, lower secondary education; 3-4, upper and postsecondary non-tertiary education; 5-6, tertiary education.

TABLE 3: Multivariate logistic regression analysis of risk factors associated with anti-hepatitis $E$ virus immunoglobulin $G$ seropositivity among liver transplant recipients.

\begin{tabular}{|c|c|c|c|}
\hline Exposure group/variables & OR & $95 \% \mathrm{Cl}$ & p \\
\hline Sex (female vs. male) & 5.65 & $2.60-12.28$ & $<0.001$ \\
\hline Sewage system (public sewer system vs. septic tank) & 3.05 & $1.39-6.66$ & 0.005 \\
\hline \multicolumn{4}{|l|}{ Educational level ${ }^{* *}$} \\
\hline ISCED 2 & 0.94 & $0.42-2.09$ & 0.886 \\
\hline ISCED 3-4 & 1.99 & $0.52-7.55$ & 0.308 \\
\hline ISCED 5-6 & 0.084 & $0.09-0.73$ & 0.005 \\
\hline
\end{tabular}

${ }^{* *}$ The International Standard Classification of Education (ISCED): 1, primary education; 2, lower secondary education; 3-4, upper and postsecondary nontertiary education; 5-6, tertiary education. 
often in contact with pigs than the urban population; therefore, the probability of exposure to $\mathrm{HEV}$ is higher in the rural population compared to the urban population.

Additionally, the use of the stream/well as a source of water was identified as a risk factor in some countries ${ }^{14}$. Approximately $14 \%$ of the population in Croatia is using drinking water from individual water wells or cisterns. In a group of liver transplant patients presented in this study, 7.7\% used a well as the source of drinking water, and $38.1 \%$ used septic tanks within the household sewage system. Both factors were found to be associated with HEV seroprevalence.

Consumption of contaminated pork products is one of the well-known risk factors for human HEV infection. Production and consumption of cured and thermally unprocessed meat and occupational exposure were associated with anti-HEV IgG seropositivity in some studies ${ }^{15}$, which was not established in our cohort of transplant patients.

This study has some limitations that need to be addressed. Determining anti-HEV IgG and IgM seroprevalence rate is important in assessing past and recent infections. However, in immunocompromised patients such as liver transplant recipients, immunosuppressive therapy may compromise humoral immune response and limit the diagnostic value of serology. In these cases, RT-PCR is required to establish an active infection. Moreover, the study included a relatively small number of patients; therefore, the results should be cautiously interpreted. Despite these limitations, this is the first study on anti-HEV prevalence in Croatian liver transplant recipients that offers new insights into HEV epidemiology in this population group.

In conclusion, the results of this study showed that anti-HEV IgG seroprevalence rate in Croatian liver transplant patients is high $(24.4 \%)$ and is consistent with the prevalence rates in other endemic regions in Europe. Older age, female gender, living in a rural area with a farm as a household setting, using a water well as a main water supply system, and using a septic tank within a household sewage system were identified as the main risk factors associated with HEV seropositivity. Further studies on a large number of SOT recipients are needed to confirm these observations and define the prevalence, clinical significance, and routes of transmission of $\mathrm{HEV}$ infection in high-risk population groups.

\section{Conflict of interest}

The authors declare that there is no conflict of interest.

\section{Financial Support}

This study was supported in part by the Zaklada Adris Grant "Hepatitis E virus infection before and after solid-solid organ transplantation in Croatia," University Hospital Merkur, Liver Transplant Centre (to Anna Mrzljak).

\section{REFERENCES}

1. Kamar N, Selves J, Mansuy JM, Ouezzani L, Péron JM, Guitard $\mathrm{J}$, et al. Hepatitis $\mathrm{E}$ virus and chronic hepatitis in organ-transplant recipients. N Engl J Med. 2008;358(8):811-7.

2. Pavio N, Meng XJ, Doceul V. Zoonotic origin of hepatitis E. Curr Opin Virol. 2015;10:34-41.

3. Buti M, Cabrera C, Jardi R, Castells L, Esteban R. Are recipients of solid organ transplantation a high-risk population for hepatitis $\mathrm{E}$ virus infection? Liver Transpl. 2010;16(1):106-7.

4. Moal V, Legris T, Motte A, Vacher-Coponat H, Fages L, JourdeChiche N, et al. Systematic serological testing for hepatitis E virus in kidney transplant recipients. J Clin Microbiol. 2015;53(5): 1523-30.

5. International Registry on Organ Donation and Transplantation Database. IRODaT. [Internet] [updated 2017 Dec; cited 2018 Mar 28]. Available from: http://www.irodat.org/img/database/pdf/ IRODaT\%20newletter\%20Final\%202016.pdf

6. Vilibic-Cavlek T, Vilibic M, Kolaric B, Jemersic L, Kucinar $\mathrm{J}$, Barbic L, et al. Seroepidemiology of hepatitis E in selected population groups in Croatia: A prospective pilot study. Zoonoses Public Health. 2016;63(6):494-502.

7. Pischke S, Suneetha PV, Baechlein C, Barg-Hock H, Heim A, Kamar $\mathrm{N}$, et al. Hepatitis $\mathrm{E}$ virus infection as a cause of graft hepatitis in liver transplant recipients. Liver Transpl. 2010;16(1):74-82.

8. Buffaz C, Scholtes C, Dron AG, Chevallier-Queyron P, Ritter J, Andre P. Hepatitis E in liver transplant recipients in the RhoneAlpes region in France. Eur J Clin Microbiol Infect Dis. 2014;33(6): 1037-43.

9. Yoon Y, Jeong HS, Yun H, Lee H, Hwang YS, Park B, et al. Hepatitis E Virus (HEV) seroprevalence in the general population of the Republic of Korea in 2007-2009: a nationwide cross-sectional study. BMC Infect Dis. 2014;14:517.

10. Verhoef L, Koopmans M, Duizer E, Bakker J, Reimerink J, Van Pelt W. Seroprevalence of hepatitis $\mathrm{E}$ antibodies and risk profile of HEV seropositivity in The Netherlands, 2006-2007. Epidemiol Infect. 2012;140(10):1838-47.

11. Hartl J, Otto B, Madden RG, Webb G, Woolson KL, Kriston L, et al. Hepatitis E Seroprevalence in Europe: A Meta-Analysis. Viruses. 2016;8(8) pii:E211.

12. Petrović T, Lupulović D, Jiménez de Oya N, Vojvodić S, Blázquez $\mathrm{AB}$, Escribano-Romero E, et al. Prevalence of hepatitis E virus (HEV) antibodies in Serbian blood donors. J Infect Dev Ctries. 2014;8(10):1322-7.

13. Lipej Z, Novosel D, Vojta L, Roić B, Simpraga M, Vojta A. Detection and characterisation of hepatitis $\mathrm{E}$ virus in naturally infected swine in Croatia. Acta Vet Hung. 2013;61(4):517-28.

14. Meng,QF, You HL, Wang WL, Zhou N, Dong W, Cong W. Seroprevalence and risk factors of hepatitis E virus infection among children in China. J Med Virol. 2015;87(9):1573-7.

15. Kamar N, Garrouste C, Haagsma EB, Garrigue V, Pischke S, Chauvet $\mathrm{C}$, et al. Factors associated with chronic hepatitis in patients with hepatitis $\mathrm{E}$ virus infection who have received solid organ transplants. Gastroenterology. 2011;140(5):1481-9. 